\title{
Beneficial Effect of Physical Activity in Hemodialysis Patients
}

\author{
K Girija ${ }^{1, *}$, R.Radha ${ }^{2}$ \\ ${ }^{1}$ Department of Food Science and Nutrition \\ ${ }^{2}$ Department of Food Service Management and Dietetics, Avinashilingam Institute of Home Science and Higher Education for Women, \\ Coimbatore \\ *Corresponding Author: girija.nutrition@gmail.com
}

Copyright (C) 2013 Horizon Research Publishing All rights reserved.

\begin{abstract}
Hemodialysis patients are significantly less active than healthy, sedentary individuals and a low intrinsic motivation has been identified as a major barrier to prescribing exercise. Regular physical activity is associated with enhanced health and well being. World Health Organisation (WHO) revealed that regular physical activity is widely recognized as a mean of preventing the occurrence of many chronic diseases and reduced risk of all-cause mortality. Physical activity during illness results in improvement in physical function. Physical activity helps dialysis patients to improve their well-being. The association of decreasing levels of leisure-time physical activity with increased mortality risk is less certain in ESRD patients than in the general population. Studies have shown that hemodialysis patients physically being active had efficient clearance of urea during dialysis compared to physically inactive patients. Yoga and exercise in hemodialysis patient increases the quality of life through reduced stress level. In our country there is lack of awareness of potential benefits of physical activity in hemodialysis patients and there are no facilities available in in-centre hemodialysis centre to implicate the exercise programe during dialysis compared to Western hemodialysis centres. So there is the great need to impart the knowledge among the patients to obtain the complete standard of living with daily physical activity during on and off dialysis days.
\end{abstract}

Keywords Hemodialysis, Physical Activity, Dialysis Adequacy

\section{Introduction}

Regular physical activity is associated with enhanced health and well being. World Health Organisation [1] revealed that regular physical activity is widely recognized as a mean of preventing the occurrence of many chronic diseases and reduced risk of all-cause mortality. Physical activity during illness results in improvement in physical function [2].

Numerous health-related benefits derived from engaging in appropriately structured exercise regimens have been documented extensively with sedentary adults, frail elderly persons, and individuals with a wide array of chronic illnesses. Chronic kidney disease is associated with dyslipidemia, which persist as kidney failure progresses and continues to affect clinical outcomes in patients on hemodialysis [3]. Hemodialysis patients are at increased risk of heart disease due to abnormalities in atherogenic lipids and apolipoproteins [4]. Physical functioning in patients with end-stage renal disease treated with dialysis is less, measured using objective laboratory measures, self-reported measures or physical performance testing [5]. Regular assessment of physical functioning and encouragement of increased physical activity are still given minimal attention within the nephrology community [6]. Rehabilitation interventions are most frequently discussed in relation to dialysis patients mostly ESRD patients along with rehabilitation programs for persons who have a kidney transplant [7-8].

\section{Physical Activity Level in Hemodialysis Patient- Global Scenario}

Hemodialysis patients are significantly less active than healthy, sedentary individuals [9] and a low intrinsic motivation has been identified as a major barrier to prescribing exercise in this cohort. Approximately 3.2 million people die each year due to lack of physical activity. People who are physically inactive have a $20 \%$ to $30 \%$ increased risk of mortality. Regular physical activity reduces the risk of cardiovascular disease including high blood pressure, diabetes mellitus, breast cancer, colon cancer, stress and depression. Insufficient physical activities are more prevalent in high-income countries and very high levels of physical inactivity are now seen in some middle-income countries especially among women. Patients with end-stage renal disease (ESRD) on dialysis have 
physical activity levels which are $20 \%-50 \%$ lower than ageand sex-matched sedentary population controls [9-10]. The reduced physical activity in hemodialysis patients was assessed by flexibility test and found that depression played a major role is preventing the hemodialysis subjects to be physically active [11]. In India hemodialysis patients are physically inactive because of their poor health condition and lack of awaremess about the health benefits of being physically active.

\section{Benefits of Physical Activity in Hemodialysis}

Physical activity helps dialysis patients to improve their well-being [12]. The association of decreasing levels of leisure-time physical activity with increased mortality risk is less certain in ESRD patients than in the general population [13]. It is therefore plausible that exercise may also lead to a corresponding decrease in morbidity and mortality rates in individuals with ESRD. An intervention with such an effect would be welcomed in the ESRD population, which is known to have high mortality rates and burden of cardiovascular disease [14].

\subsection{Exercise and Clearance}

Recent renewed interest in activity and exercise as an important component of dialysis treatment is encouraging. The positive impact of dialysis exercise programmes on people's physical functions was recently demonstrated [15]. Parson [16] reported that exercises during dialysis enhanced dialysate urea removal but not serum urea clearance. Alterations in the timing of exercise during dialysis and modality may be required to elicit increases in serum urea clearance. It is recommended that mild exercise during dialysis should be performed during the first two hours of dialysis.

Bennet [17] proposed the formula to determine the Dialysis Exercise Adequacy (DEA) that takes into account the frequency $(\mathrm{F})$ and duration $(\mathrm{T})$ of the exercise in hours and the age (A) of the person in years; thus DEA=FTA/100. A minimum DEA "target" could be established (for example 1.0) that considers the amount and frequency of exercise relative to the age of the person. Few studies have measured the acute or long-term effects of intradialytic exercise on urea removal and dialysis efficacy in vivo. In response to a single bout of exercise, dialysis efficacy has been shown to increase between $15 \%$ and $25 \%$ [18-19].

Cycling exercise before or during dialysis is an option in some treatment centers. Training of exercise during dialysis treatments may improve solute removal by increasing blood flow to muscle and efflux of urea and other toxins into the vascular compartment where they can be removed [20]. Moreover there is a possibility of reduced exercise tolerance during dialysis resulting from electrolyte and fluid shifts and exercise could lead to dialysis-associated hypotension [21].
It appears; however, that exercise is generally well tolerated within the first 1-2 hours of a hemodialysis (HD) session [22]. Also, the decrease in $\mathrm{C}$ - reactive protein (CRP) is an interesting finding. CRP is connected to residual renal function [23]. The reduced inflammatory process in the dialysis patients becomes better after six months of repeated cycle training and this might be an adequate prescription to be followed by every patient on hemodialysis.

Zaluska [24] reported a $16 \%$ improvement in dialysis efficacy in 10 hemodialysis patients who cycled for 30 minutes during the first hour of dialysis over 6 months; however, some of this improvement may have been related to a significant increase in protein catabolic rate, which would augment urea clearance. So far no study has demonstrated the effectiveness of intradialytic exercise program can result in improved serum urea clearance to determine dialysis efficacy [25].

\subsection{Physiological Benefits of Exercise for Hemodialysis Patients}

Studies had shown that dialysis patients are debiliated due to anemic condition. Hemodialysis patients reported low levels of physical activity which was determined using questionnaire. Muscle defect due to uremic condition, hyperparathyroidism, hormonal imbalance, resistance to growth hormone and muscle atrophy may be the reason for reduced physical activity. Exercise training in ESRD has lessened the impact of ramifications by the attenuation of uremic neuropathies and myopathies, improved cardiac function, reduced blood pressure, increased physical work capacity, and overall enhancement in health related quality of life [26]. Although most exercise programs have been instituted between dialysis sessions, recent investigations have promoted the concept of intradialytic exercise as a convenient intervention to improve compliance, provide motivation in a structured environment, and facilitate the medical monitoring of the exercising patient [27-28].

A large research literature documents a variety of potential benefits that ESRD patients may achieve from exercise training [21]. Kutner [29] quoted the evidence from other studies that exercise training for ESRD patients helps the improvement in arterial stiffness [30], decrease in pulse pressure [16], increased aerobic capacity [21], reduced need for antihypertensive medications [31], increase in concentration of hemoglobin and hematocrit levels and improved lipid metabolism [32]. Dialysis patients at rest do not show significant differences in their muscle blood flow compared to control subjects; nevertheless, their muscle blood flow rises significantly less than in control subjects during both submaximal and maximal exercise [33]. In a systematic review of 29 clinical trials, it was shown that physical condition was significantly improved following the use of aerobic training [34].

Trisha et al. [25] reported that intradialytic exercise from a physiologic perspective with the hypothesis that the increased muscle blood flow and greater amount of open 
capillary surface area in working muscles will result in a greater flux of urea and associated toxins from the tissue to the vascular compartment for subsequent removal at the dialyser. Goldberg et al [32] examined the metabolic effects of exercise training in hemodialysis patients and reported that training lowered triglycerides and increased HDL cholesterol. Numerous studies in both the general and dialysis population lend circumstantial evidence and point to plausible physiological mechanisms for a morbidity and survival benefit with improved physical fitness and function and increasing physical activity levels in patients with ESRD [35]. The exercise increases the insulin sensitivity in the skeletal muscle and decreases the insulin in the blood which suggests less adiposities and healthy lipid profile with increased HDL-cholesterol and decreased triglycerides. Due to this, testosterone levels have been found to increase and androgen receptors which increase the effect of testosterone [36].

Studies of the effects of exercise on VO2 peak have provided important information because they showed that patients with kidney disease could respond physiologically to exercise training in a manner that is similar to other patient groups. However, qualified success of severe aerobic exercise training that is designed to increase $\mathrm{VO} 2$ peak should be considered. The patients who are active have generally been the healthiest individuals who receive hemodialysis, usually a small percent of available patients [37], and it is not clear that more typical (i.e., less healthy) patients with kidney disease will be willing or able to undergo vigorous exercise training. Orti [38] reported that moderate evidence exists on the improvement on capacity of exercise on aerobic training, combined or isolated with strength training. Strength training improves health related quality of life and has positive effect on functional capacity and improves lower limb strength.

\subsection{Psychological Benefits of Physical Activity in Hemodialysis Patients}

Mental health needs, especially debilitating levels of depressed mood and clinical depression, are prevalent among renal patients especially those who are underdoing dialysis [39]. Antidepressants are frequently advised, but negative side effect and drug interactions of antidepressants are of concern [40]. Yaghmayi [41] quoted that chronic renal failure has mutual effects on physical, psy-chological and functional status of individu-als which causes types of deprivation and lifestyle changes including financial problems, unemployment, change in familial roles, restriction in fluid intake and diet and reduction in achieving long term goals. Stress is directly associated with the risk of chronic renal failure such as hypertension. Further-more, other studies have pointed out that stress is correlated with low socioeconomic status and such conditions can be a risk factor for progression of chronic renal disease through association with other socio-mental factors and behaviors such as alcohol consumption, smoking and drug use [42].
Exercise training may have positive benefits on other factors that are important clinical issues in dialysis patients, including oxidative stress, cardiovascular risk profile and inflammation [5]. Nancy et al [43] reported that screening and management of depressive symptoms and support for increased activity level may facilitate patients' opportunity for continued employment after initiation of dialysis and improving their quality of life.

Fatouros et al [44] implies that hemodialysis patient demonstrate higher elevated oxidative stress levels and a lower antioxidant status than healthy controls at rest and following exercise. Acute exercise appears to exacerbate elevated oxidative stress response in hemodialyzed patients probably due to diminished defence by antioxidants. However, increased aerobic capacity helps to decrease oxidative stress in this population.

Tayyebi et al. [45] in reported that implementation of yoga exercise, especially Hatha yoga as a combination of breathing, positional, and relaxing exercises, along with music as well as Quranic and religious recitation left significant impact on these patients. These effects have each been approved in various studies; in a study by Hojjat et al. [46] to investigate the effect of music and sounds of Quran on dialysis adequacy among hemodialysis patients, the mean dialysis adequacy was significantly increased, and more interestingly, the sounds of Quran played more effective role in such an augmentation [46]. NCD (National Cooperative Dialysis) study has also proved that the more the adequacy of dialysis is, the less the effects of uremia on body different systems and patients mortality will be decreased [47]. The close relationship between mind and body has long been clear and the goal of yoga as an Eastern philosophy of life was to bring the balance between the two, which affects the overall sense of health and augments the quality of life through enhancing the compatibility of psychological, neurological, immune, and cognitive systems and modulating the immune system [48].

\section{Conclusion}

Bennett et al. [17] recently reviewed the literature to identify elements needed to sustain inclusion of exercise in routine care and/or as a normal part of the individual's daily life, whether performed on or off dialysis. In India, most of the hemodialysis centres do not include exercise as the part of the treatment compared to Western hemodialysis centres. Since the hemodialysis patients are both physiologically and psychologically ill, knowledge on physical activity helps in improving their overall well being. Health care workers such as physicians, technician, Renal Dietician, nurses should play a major role in educating the patients on importance of physical activity. 


\section{REFERENCES}

[1] World Health Organization. World Health Report (2002). Reducing risks, promoting healthy lifestyle. World Health Organization, Geneva pp. 61, 218-219, 226-227. Retrieved in March 2007

http://www.who.int/whr/2002/en/index.html.

[2] A Stewart, R Hays, K Wells, et al. Long term functioning and well being outcomes associated with physical and exercise in patients with chronic conditions in the medical outcomes study. J Clin Epidemiol. Vol 47, 719-730, 1994.

[3] E Kimak and J Solski. ApoA- and apoB-containing lipoproteins and $\mathrm{Lp}(\mathrm{a})$ concentration in non-dialyzed patients with chronic renal failure, Renal Failure, Vol. 24, No. 4, 485-492, 2002

[4] S.S. Prichard. Impact of dyslipidemia in end-stage renal disease, Journal of the American Society of Nephrology, Vol.14, No.9, supplement 4, S315-S320, 2003

[5] Patricia Painter. Physical functioning in end stage renal disease patients: Updates 2005. Hemodialysis International. Vol 9, No.3, 218-235, 2005.

[6] P Painter. Physical functioning in CKD; it's time to GET MOVING! Dialysis Times News and Views from Renal Research Institute, Vol 17, No. 2, 2, 2010.

[7] E.J.Gordon, T. Prohaska, L.A.Siminoff, P.J. Minich, A.R. Sehgal. Needed: tailored exercise regimens for kidney transplant patients, American Journal of Kidney Diseases, Vol.45, 769-774, 2005

[8] H.Müller, W. Gwinner, H.Haltenhof, J. Kornhuber, J.M. Maler. Psychological risk factors of kidney transplant patients, Kidney International, Vol.77, 833, 2010

[9] K.L. Johansen, G.M. Chertow, A.V.Ng, et al. Physical activity levels in patients on hemodialysis and healthy sedentary controls, Kidney Int, Vol. 57, 2564-2570, 2000.

[10] A.M. O'Hare, K. Tawney, P. Bacchetti, K.L. Johansen. Decreased survival among sedentary patients undergoing dialysis: results from the Dialysis Morbidity and Mortality Study Wave 2, Am J Kidney Dis, Vol. 41, 447-454, 2003.

[11] R.M. Carney, R.D. Wetzel, J. Hagberg and A.P. Goldberg. The relationship between depression and aerobic capacity on hemodialysis patients, Psychosom Med, Vol. 48, 143-147, 1986.

[12] WHO, Diet, nutrition and the prevention of chronic diseases, World Health Organisation: Geneva, 2003

[13] D.E. Warburton, C.W. Nicol and S.S Bredin. Health benefits of physical activity: the evidence, CMAJ, Vol. 174, 801-809, 2006.

[14] Canadian Institute of Health Information. Five-year survival in ESRD patients on dialysis, with or without diabetes, by age, 2003-2007 period survival. 2009. Available at:http://secure.cihi.ca/cihiweb/dispPage.jsp?cw_page=statist ics_results_source_corr_e. Accessed Jan 27, 2010.

[15] N. Smart and M. Steele. Exercise training in haemodialysis patients: A systematic review and meta-analysis. Nephrology (Carlton), Vol. 16, No.7, 626-632, 2011

[16] T.L. Parsons, E.B. Toffelmire, C.E. King-VanVlack. The effect of an exercise program during hemodialysis on dialysis efficacy, blood pressure and quality of life in end-stage renal disease (ESRD) patients, Clin Nephrol. Vol.61, No.4, 261-74, 2004.

[17] P.N. Bennett, L. Breugelmans, R. Barnard, M. Agius, D.Chan, D. Fraser, L. McNeill, L. Potter. Sustaining a hemodialysis exercise program: a review, Seminars in Dialysis, Vol. 23, No.1, 62-73, 2012

[18] C.H. Kong, J.E. Tattersall, R.N. Greenwood and K Farrington. The effect of exercise during haemodialysis on solute removal, Nephrol Dial Transplant, 14:2927-31, 1991

[19] Y.Sun, B. Chen, Q. Jia, J. Wang. The effect of exercise during hemodialysis on adequacy of dialysis, [Chinese], Zhonghua Nei Ke Za Z, Vol. 41, 79-81, 2002.

[20] T.L. Parsons, E.B. Toffelmire, C.E. King-VanVlack. Exercise training during hemodialysis improves dialysis efficacy and physical performance, Archives of Physical Medicine and Rehabilitation, Vol. 87, 680-687, 2006

[21] K.L. Johansen. Exercise in the end-stage renal disease population, Journal of the American Society of Nephrology, Vol.18, 1845-1854, 2007.

[22] P. Painter, G. Moore, L. Carlson, S. Paul, J. Myll, W. Phillips, W. Haskell. Effects of exercise training plus normalization of hematocrit on exercise capacity and health-related quality of life, American Journal of Kidney Diseases, Vol.39, 257-265, 2002.

[23] R. Pecoits-Filho, O. Heimburger, P. Barany, M. Suliman, I. Fehrman-Ekholm, B. Lindholm and P.Stenvinkel. Associations between circulating inflammatory markers and residual renal function in CRF patients. American Journal of Kidney Diseases, Vol 41, No.6: 1212-1218, 2003.

[24] Zaluska, W.T. Zaluska, A. Bednarek-Skublewska, A. Ksiazek A. Nutrition and hydration status improve with exercise training using stationary cycling during hemodialysis (HD) in patients with endstage renal disease (ESRD), Ann Univ Mariae Curie Sklodowska, Vol.57, 342-346, 2002.

[25] L. Trisha, P. Parsons, B. Edwin, Toffelmire, E. Cheryl, King-VanVlack. Exercise Training During Hemodialysis Improves Dialysis Efficacy and Physical Performance, Arch Phys Med Rehabil, Vol.87, 680-688. 2006.

[26] P. Painter, L. Carlson, S. Carey, S.M. Paul, J. Myll. Low-functioning hemodialysis patients improve with exercise training, Am J Kidney Dis, Vol.36, 600-608, 2000.

[27] P. Painter, L. Carlson, S. Carey, S.M.Paul, J. Myll. Physical functioning and health-related quality-of-life changes with exercise training in hemodialysis patients, Am J Kidney Dis, 35:482-92, 2000

[28] B.S. Cheema, B.C. Smith and M.A. Singh. A rationale for intradialytic exercise training as standard clinical practice in ESRD, Am JKidney Dis, Vol. 45, 912-6, 2005.

[29] N.G. Kutner. Kidney disorders: end stage renal disease/dialysis. In: JH Stone, M Blouin, editors. International Encyclopedia of Rehabilitation. Available online: http://cirrie.buffalo.edu/encyclopedia/en/article/284/, 2012.

[30] S. Mustata, C. Chan, V. Lai, J.A.Miller. Impact of an exercise program on arterial stiffness and insulin resistance in hemodialysis patients, Journal of the American Society of 
Nephrology, Vol.15, 2713-2718, 2004.

[31] B.W. Miller, C.L. Cress, M.E.Johnson, D.H. Nichols, M.A.Schnitzler. Exercise during hemodialysis decreases the use of antihypertensive medications, American Journal of Kidney Disease, Vol.39, 828-833, 2002

[32] A.P. Goldberg, J.M. Hagberg, J.A. Delmez et al. Metabolic effects of exercise training in hemodialysis patients, Kidney International, Vol.18, No. 6, 754-761, 1980

[33] J.R. Bradley, J.R. Anderson, D.B. Evans, A.J. Cowley. Impaired nutritive skeletal muscle blood flow in patients with chronic renal failure. Clin Sci (Lond), Vol.79: 239-245, 1990.

[34] B.S.B. Cheema, M.A. Fiatarone Singh. Exercise training in patients receiving maintenance hemodialysis: a systematic review of clinical trials, Am J Nephrol, Vol.25, 352-364, 2005

[35] Bohm et al. Exercise therapy for ESRD, Journal of Nephrology, Vol. 23, No.3, 235-243, 2010.

[36] W.J. Kraemer and N.A. Ratamess. Hormonal responses and adaptations to restistance exercise and training, Sports Med, Vol.35, 339-61, 2005

[37] M.R. Suh, H.H. Jung, S. B. Kim, J.S. Park, W.S. Yang. Effects of regular exercise on anxiety, depression, and quality of life in maintenance hemodialysis patients. Ren Fail, Vol.24, $337-345,2002$

[38] E. Segura-Ortí. Exercise in haemodyalisis patients: a literature systematic review, Nefrologia. Vol.30, No.2, 236-46, 2010.

[39] F.O. Finkelstein, D. Wuerth, L.K. Troidle, S.H. Finkelstein. Depression and end-stage renal disease: a therapeutic challenge, Kidney International, Vol.74, 843-845, 2008

[40] J. Chilcot, D. Wellsted, K. Farrington. Depression in end-stage renal disease: current advances and research, Seminars in Dialysis, Vol. 23, No.1, 74-82, 2010

[41] F. Yaghmayi, E. Khalfi, N. Khost, A. Alavi. The rela-tionship between self- concept dimensions of helth status in pateints treated with hemodialyses in med-ical sience hospital in 2004, Pajoohandeh Journal, Vol. 10, No. 6, 9-15, (Persian). 2005.

[42] K.R. Tuttle, D. Sunwold and H.Kramer. Can comprehen-sive lifestyle change alter the course of chronic kid-ney disease?, SeminNephrol, Vol.29, No.5, 512-23, 2009

[43] G. Nancy, Kutner, Rebecca Zhang, Yijian Huang, and L. Kirsten, Johansen. Depressed Mood, Usual Activity Level, and Continued Employment after Starting Dialysis, Clin J Am Soc Nephrol. November, Vol. 5, No. 11, 2040-2045, 2010

[44] I.G. Fatouros, P. Pasadakis, A. Sovatzidis, A. Chatzinikolaou, S. Panagoutsos, D.Sivridis, I. Michailidis, I. Douroudos, K. Taxildaris, V. Vargemezis. Acute exercise may exacerbate oxidative stress response in hemodialysis patients, Nephron Clic Pract., Vol.109, No.2, c55-64, 2008.

[45] Ali Tayyebi, Malihe Babahaji, Mahdi Sadeghi Sherme, Abbas Ebadi, Behzad Eynollahi. Study of the effect of Hatha Yoga exercises on dialysis adequacy, Iranian Journal of Critical Care Nursing, Vol. 4, No.4, 183 - 190, 2012.

[46] M. Hojat, Z. Zohadat Pour, M. Nasr-Esfahani. Sound effects compared with the conditions of silence Quran, Arabic and Persian music on the adequacy of hemodialysis, J Special Nursing, Vol. 3, No.2, 69-74, 2010.

[47] A. Raisifar, M. Torab pour, P. Mohseni Zad. Review adequacy of dialysis in hemodialysis patients referred to the center city of Abadan in the 2006, Vol. 2, No.4, 157-60, 2009.

[48] M.S. Garfinkel, A. Singhal, W.A. Katz, D.A. Allan, R. Reshetar, H.R. Schumacher Jr. Yoga-based intervention for carpal tunnel syndrome: a randomized trial, JAMA, Vol.280, No.18, 1601-3, 1998. 